\title{
116. Differential Staining of Chromosomes of Two Salmonoid Species, Salvelinus leucomaenis (Pallas) and Salvelinus malma (Walbaum) ${ }^{1)}$
}

\author{
By Syuiti Abe and Jun-ichi Muramoto
}

(Comm. by Sajiro Makıno, M. J. A., Sept. 12, 1974)

Currently developed staining procedures have proved useful for distinguishing individual chromosomes or chromosomal segments by recognizably differentiated banding patterns. The application of new staining techniques to piscine chromosomes has not yet been very efficient for the characterization of chromosomes. Very recently, Howell and Bloom (1973) demonstrated certain sex-associated fluorescent bodies in the chromosomes and spermatozoa of mudminnow after quinacrine staining.

With a hope to obtain morphological criteria on the chromosomal relationship between two closely related species of trouts, Salvelinus leucomaenis and S. malma, we have attempted some banding analyses of somatic chromosomes.

Materials and methods. Tissues were aseptically removed from the kidney, spleen, air bladder, and testis of S. leucomaenis ( 2 females and 1 male) and S. malma (1 female, 1 male and 1 sex-unknown fry). They were washed in a saline solution containing penicillin (120 $\mathrm{IU} / \mathrm{ml})$ and kanamycin $(100 \mu \mathrm{g} / \mathrm{ml})$, and minced with scissors. Aliquots of the tissue fragments suspended in $5 \mathrm{ml}$ of Eagle's minimum essential medium with $20 \%$ fetal calf serum were seeded into $30 \mathrm{ml}$ Falcon plastic flasks, and cultured at $19 \pm 1^{\circ} \mathrm{C}$ for $20-28$ days. Six to twelve hours before harvest, colcemid $(10 \mu \mathrm{g} / \mathrm{ml}, 1$ or 2 drops) was added to the medium. Air dried chromosome preparations were made following hypotonic pretreatment $(0.075 \mathrm{M} \mathrm{KCl}$ for $20 \mathrm{~min}$., at room temp.) and fixation with 3:1 methanol-acetic acid. The Q-staining (Caspersson et al. 1970), C-staining (Sumner 1972), and G-staining (Sumner et al. 1971) procedures were applied for demonstrating chromosome bands.

Results and remarks. The diploid chromosome numbers were 82 in S. malma and 84 in S. leucomaenis. The karyotype of S. malma consisted of 16 meta- or submetacentrics, 4 subtelocentrics and 62 acrocentrics, while that of $S$. leucomaenis was characterized by

1) Contributions from the Chromosome Research Unit, Faculty of Science, Hokkaido University, Sapporo 060. 
having 4 additional acrocentrics and by missing 2 subtelocentrics.

The Q-stained metaphase chromosomes in both species were characterized by rather faint and poorly differentiated fluorescence, except the centromeric and/or telomitic regions of certain chromosomes showing a higher fluorescence intensity (Figs. 1 and 2). At least, 14 pairs of acrocentrics of $S$. leucomaenis had a bright telomitic band in each. Among them, one medium sized pair was outstanding by having a very large and bright fluorescent block in the distal region. In S. leucomaenis the Q-positive centromeric band was noted only in one pair of acrocentric elements, while in S. malma all 8 pairs of meta- or submetacentrics and at least 16 pairs of acrocentrics had an apparently bright centromeric band in each. The centromeric Qband was very apparent in 2 medium-sized acrocentric pairs of the latter species. In $S$. malma, some 14 pairs of acrocentrics had a telomitic band, among which 9 pairs showed both telomitic and centromeric bands.

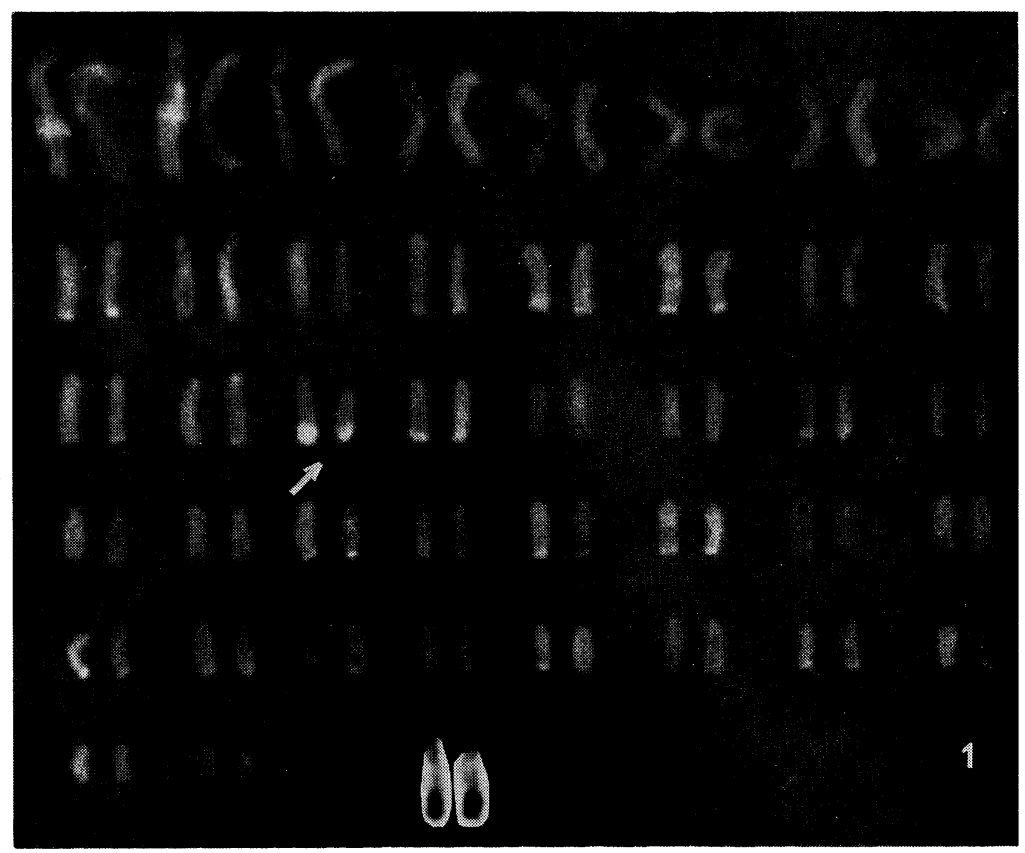

Fig. 1. Q-stained karyotype of S. leucomaenis (male). Arrow indicates an acrocentric pair with a large bright telomitic band. The corresponding C-stained chromosomes are shown in the bottom row.

Thus, the presence or absence of centromeric Q-band in certain biarmed elements served to the karyotypic distinction between the two species. Furthermore, the entire short arm involving the centromeric region of one pair of submetacentrics of S. malma was much 
brighter than the negatively fluorescent long arm. No such distinctive feature was observed in any biarmed element of S. leucomaenis. No sex-associated fluorescent patterns as those reported in the mudminnow by Howell and Bloom (1973) were noted in the present species.

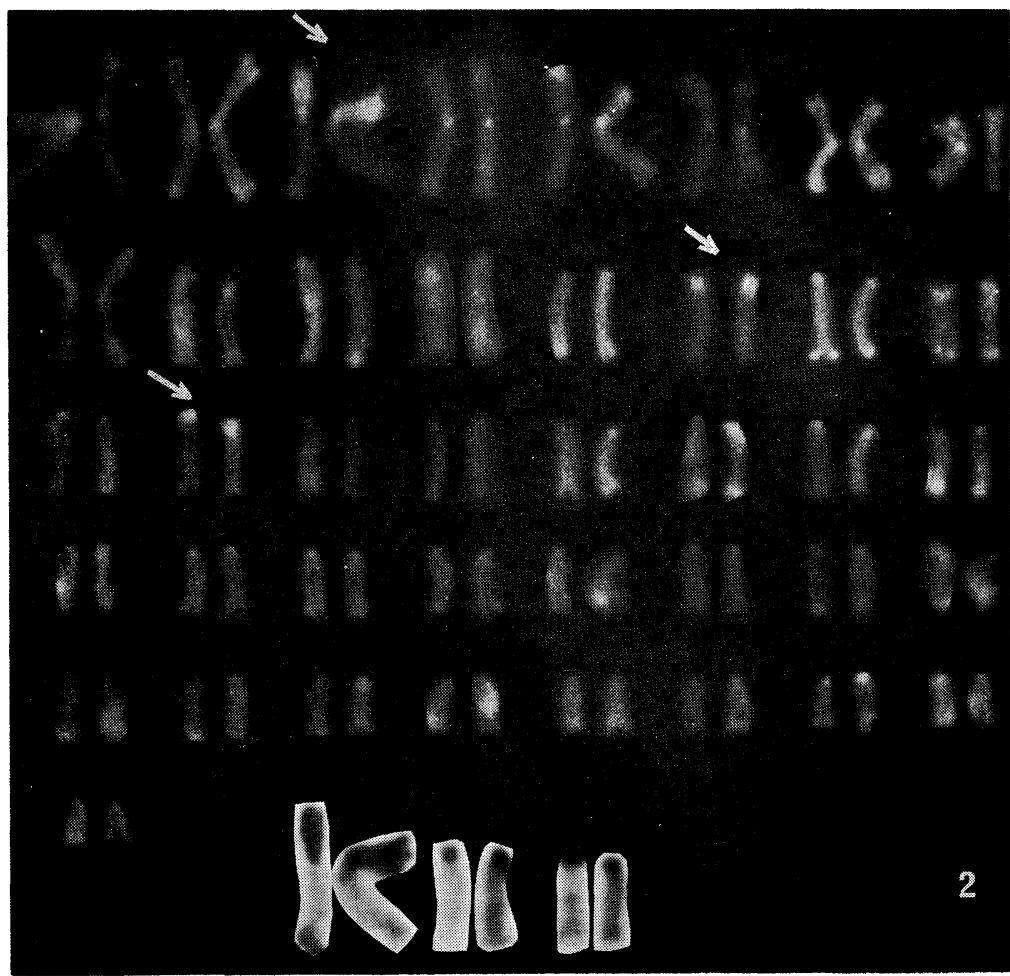

Fig. 2. Q-stained karyotype of S. malma (female). Three pairs of chromosomes with conspicuous heterochromatin after C-banding are shown in the bottom row. The corresponding pairs are arrowed in the Q-stained karyotype.

In contrast to the Q-band pattern, the G-banding process yielded much less informative feature, since there was no chromosomes which had characteristic and consistent banding patterns. Since the ASG method after Sumner et al. (1971) was employed, the optimal condition involved in the denaturation-renaturation process might be different between mammals and fishes. Though other physico-chemical factors should also be considered for the underlying mechanism, further technical improvement may be needed for the G-banding method of the fish chromosomes. An attempt with the trypsin banding technique (Seabright 1971) was made for this purpose in some fish chromosomes, but the results were unsatisfactory. 
The BSG technique after Sumner (1972) for the C-bands produced quite satisfactory results. The locations of constitutive heterochromatin of the chromosomes as revealed by the Sumner's C-staining method were compatible with the Q-band positive regions (Figs. 1 and 2). A large heterochromatic block on the short arm of a submetacentric pair and centromeric heterochromatin in about a half of the acrocentric pairs as well as in most biarmed pairs were useful for prominent markers for S. malma. On the other hand, a pair of medium-sized acrocentrics with a large telomitic C-band characterized the karyotype of S. leucomaenis. The similarity between C- and Q-band patterns as seen in the present species has been known in some plant species, for instance in Rhoeo discolor (Natarajan and Natarajan 1972) and Secale cereale (Sarma and Natarajan 1973). In human chromosomes, C-band positive regions are not strictly comparable to the Q- and G-band positive ones, and also to certain Cbands; generally those on chromosomes Nos. 1, 9 and 16 and the $Y$ chromosome have been shown to be variable (Paris Conference 1971). The situation is similar to the mouse chromosomes in which Cor G-band positive centromeric regions are Q-band negative (Committee on Standardized Genetic Nomenclature for Mice 1972). The occurrence of C-band polymorphism has been reported in certain inbred strains and natural populations of mice (Forejt 1973). Interspecific as well as intraspecific variabilities in staining properties of constitutive heterochromatin have been known to occur in some other mammalian species (Bradshaw \& Hsu 1972; Schnedl 1972; Mori et al. 1973). The evolutional implication of the diversity of the C-band heterochromatin has remained obscure. No such a polymorphism has so far been detected in our material. Further studies have been going on with particular reference to meiotic and mitotic mechanism in allied species and hybrids.

Summary. The Q-band and C-band patterns of chromosomes are investigated in two species of the Salmonidae, Salvelinus leucomaenis $(2 \mathrm{n}, 84)$ and $S$. malma $(2 \mathrm{n}, 82)$. The two species differ by the numbers of acrocentrics and subtelocentrics. The Q-banded chromosomes of both species showed rather faint and poorly differentiated fluorescence, except for brighter centromeric and/or telomitic regions of certain chromosomes. Most of Q-band positive regions in both species correspond to the location of constitutive heterochromatin as revealed by the C-staining procedure. No sexual dimorphism is observed in terms of both the Q- and C-band patterns here concerned.

Acknowledgments. We acknowledge with heartful gratitude Professor Motomichi Sasaki for his guidance and revision of the 
manuscript. We wish to express our cordial thanks to Emeritus Professor Sajiro Makino for his continuous encouragement with valuable advice. We are also grateful to Dr. Tatsuo Kubo (Faculty of Fisheries, Hokkaido University) and Mr. Hideshige Komiyama (Faculty of Agriculture, Hokkaido University) for generously supplying the materials. This work was supported by grants from the Ministry of Education.

\section{References}

Bradshaw, W. N., and T. C. Hsu (1972). Cytogenetics, 11, 436-451.

Caspersson, T., L. Zech, C. Johansson, and E. J. Modest (1970) : Chromosoma (Berl.), 30, 215-227.

Committee on Standardized Genetic Nomenclature for Mice (1972): J. Hered., 63, 69-72.

Forejt, J. (1973) : Chromosoma (Berl.), 43, 187-201.

Howell, W. M., and S. E. Bloom (1973): Nature, 245, 261-263.

Mori, M., M. Sasaki, and N. Takagi (1973) : Japan. J. Genet., 48, 381-383.

Natarajan, A. T., and S. Natarajan (1972) : Hereditas, 72, 323-330.

Paris Conference (1971): Birth Defects: Org. Art. Ser., 8, 1-46 (1972).

Sarma, N. P., and A. T. Natarajan (1973) : Hereditas, 74, 233-238.

Schnedl, W. (1972) : Chromosoma (Berl.), 38, 319-328.

Seabright, M. (1971) : Lancet, ii, 971-972.

Sumner, A. T., H. J. Evans, and R. A. Buckland (1971): Nature New Biol., 232, 31-32.

Sumner, A. T. (1972) : Exptl. Cell Res., 75, 304-306. 